\title{
BMJ Open Towards a global framework for assessing male involvement in maternal health: results of an international Delphi study
}

\author{
Anna Galle (D , ${ }^{1}$ Sally Griffin, ${ }^{2}$ Nafissa Osman, ${ }^{3}$ Kristien Roelens, ${ }^{4}$ \\ Olivier Degomme ${ }^{1}$
}

To cite: Galle A, Griffin S, Osman N, et al. Towards a global framework for assessing male involvement in maternal health: results of an internationa Delphi study. BMJ Open 2021;11:e051361. doi:10.1136/ bmjopen-2021-051361

- Prepublication history and additional supplemental material for this paper are available online. To view these files, please visit the journal online (http://dx.doi.org/10.1136/ bmjopen-2021-051361).

Received 17 March 2021 Accepted 18 August 2021

\section{Check for updates}

(C) Author(s) (or their employer(s)) 2021. Re-use permitted under CC BY-NC. No commercial re-use. See rights and permissions. Published by BMJ.

${ }^{1}$ Department of Public Health and Primary Care, Ghent University, Gent, Belgium ${ }^{2}$ International Centre for Reproductive Health-

Mozambique (ICRHM), Maputo, Mozambique

${ }^{3}$ Department of Obstetrics and Gynecology, Eduardo Mondlane University, Maputo, Mozambique ${ }^{4}$ Department of Human Structure and Repair, Ghent University, Gent, Belgium

Correspondence to

Dr Anna Galle;

anna.galle@ugent.be

\section{ABSTRACT}

Purpose Currently, no standard instrument exists for assessing the concept of male involvement in maternal health, hampering comparison of results and interpretation of the literature. The aim of this study was to construct the key elements of a global multidimensional male involvement framework, based on the latest evidence and input of experts in the field.

Methods For this purpose, a Delphi study, including an international panel of 26 experts, was carried out. The study consisted of three rounds, with $92 \%$ of respondents completing all three surveys. Experts were asked to rate indicators within six categories in terms of validity, feasibility, sensitivity, specificity and context robustness. Furthermore, they were encouraged to clarify their rating with open text responses. Indicators were excluded or adapted according to experts' feedback before inclusion. A $85 \%$ agreement was used as threshold for consensus. Results A general consensus was reached for a global framework for assessing male involvement in maternal health, consisting of five categories: involvement in communication, involvement in decision-making, practical involvement, physical involvement and emotional involvement.

Conclusions Using the male involvement framework as a tool to assess the concept of male involvement in maternal health at local, national, and international levels could allow improved assessment and comparison of study findings. Further research is needed for refining the indicators according to context and exploring how shared decision-making, gender equality and women's empowerment can be assessed and facilitated within male involvement programmes.

\section{BACKGROUND}

The important role of men in reproductive health received major global attention for the first time during the International Conference on Population and Development (ICPD) conference in $1994 .^{1}$ The language of the ICPD statement on men's role was progressive for that time, emphasising the need for equity in gender relations, with a special focus on men's shared responsibility

\section{STRENGTHS AND LIMITATIONS OF THIS STUDY}

$\Rightarrow$ A strength of the study is the translation of results into a novel and practical framework for assessing male involvement in maternal health.

$\Rightarrow$ The balanced mix of quantitative and qualitative data in this Delphi study allowed nuanced results and a rich discussion.

$\Rightarrow$ Pregnant women and their partners were not involved in the development of the framework, which is a limitation.

$\Rightarrow$ Due to its novelty, the framework might need adaptations based on new insights and evidence in the future, which can be considered as a limitation.

$\Rightarrow$ This Delphi study was conducted in a short and intense time span to guarantee a high response rate, consequently the short time span did not allow a real-time virtual or in-person meeting with the experts.

and active involvement to promote reproductive and sexual health. Signatories of the agreement believed that if men are involved in a wide range of reproductive health services in such a way that they are supported as equal partners and responsible parents, as well as clients in their own right, better outcomes in reproductive health outcomes (for themselves, mothers and newborns) can be expected. ${ }^{2}$ Since ICPD, there has been an increase in male involvement (MI) research globally, mostly with the ultimate aim of improving maternal and newborn health outcomes. While the issue has been studied in all continents, a recent systematic review found that the research angle is often different according to the context ${ }^{3}$. While most African research focuses on MI in the prevention of HIV transmission from mother to child, Western countries focus more on the role of the male partner in improving psychosocial health, while Asian countries have conducted more research with respect 
to men's role in preventing malnutrition in pregnant women and infants. Although the rationale for involving men in maternal health $(\mathrm{MH})$ is different according to the context, $\mathrm{MI}$ in $\mathrm{MH}$ is considered as an important, but often overlooked, strategy for improving maternal and newborn health outcomes worldwide. ${ }^{45}$

The perceived benefits of involving men in $\mathrm{MH}$ have been described by several systematic reviews and studies. ${ }^{4-9}$ A systematic review by Yargawa and Leonardi-Bee ${ }^{4}$ showed that MI has a beneficial impact on $\mathrm{MH}$ through reduced odds of maternal depression and improved utilisation of MH services, specifically by higher rates of skilled birth attendance and postnatal care (PNC). ${ }^{4}$ MI was also associated with decreased likelihood of childbirth complications, although the evidence was less strong. ${ }^{4}$ Noteworthy is that MI was defined differently across the included studies, with some authors focusing on male participation in healthcare services (mostly antenatal care (ANC)) and others on decision-making and financial support. ${ }^{4}$ A later review in 2018 supported the findings of Yargawa and Leonardi-Bee ${ }^{4}$ by showing that interventions to engage men were associated with improved ANC attendance, skilled birth attendance, facility birth, postpartum care, birth and complications preparedness and maternal nutrition. ${ }^{8}$

The literature shows consensus regarding the importance and benefits of involving men in $\mathrm{MH}$, but the definitions of $\mathrm{MI}$ in $\mathrm{MH}$ vary worldwide and are inconsistent. ${ }^{10}$ Some authors have defined MI as male presence at ANC and others as a combination of financial support, shared decision-making and participation in $\mathrm{MH}$ care services. ${ }^{11-13}$ The interventions to involve men also vary, ranging from inviting men for HIV testing during ANC by invitation letters to multicomponent community interventions addressing women's health and gender roles. ${ }^{12} 1415$

Criticism has grown regarding the use of single measures for assessing MI, because they often tend to focus on instrumental support (by focusing on a single act such as ANC attendance or HIV testing), which might be affected by several barriers and consequently not represent true involvement of men. ${ }^{16}$ Several authors highlight that a multiple measure is more appropriate because this allows measurement of different aspects of MI, taking into account both emotional and instrumental support. ${ }^{17} 18$ Furthermore, qualitative research has shown that the core elements of MI are very similar worldwide, whereby emotional, practical and physical support are key aspects of MI, ${ }^{19-21}$ making it likely that a global measure would be useful and feasible. On the other hand, studies also show that MI is a context-specific concept, influenced by social and cultural norms, as well as health system factors and policies. ${ }^{4223}$ Consequently, any global measurement or framework for assessing MI in $\mathrm{MH}$ will be challenged by context-specific factors and interests.

Based on our research experience regarding MI in $\mathrm{MH}$ and discussions with key stakeholders (including researchers, clinicians, women and their partners, and decision-makers), we concluded that there is a lack of a multidimensional MI index for use worldwide. While some indices have been published, they seem to have been developed for specific time-limited programmes and are not based on global evidence and/or input of experts. ${ }^{24-26}$ Consequently, it is challenging to monitor the trends in MI publications, lessons learnt, interventions and guidelines, which hamper sustainable inclusion of men in MH worldwide. While a globally standardised list of indicators could be too ambitious at this early stage, an initial exploratory study regarding an international MI framework could pave the path for a more validated measurement of MI in different programmes worldwide. Furthermore, such an instrument could allow improved comparison of findings across different contexts and facilitate the set-up of multicountry studies. Based on the research gap regarding a common set of indicators for assessing MI in MH, as well as the concerns of researchers and experts worldwide, the present study aims to retrieve a list of the most validated and important indicators of MI globally.

\section{METHODS}

\section{Delphi technique}

The Delphi technique is a method that aids in structuring a group communication process and allows participants to deal with an intricate problem as a group. ${ }^{27-29}$ The Delphi technique has numerous advantages including simplicity of implementation, enabling collection of opinions of a large array of participants with distinct expertise located in various geographical locations, while ensuring anonymity during the process. ${ }^{29}$ For the purpose of this study, a multidisciplinary panel of experts was identified and engaged in prioritising and selecting indicators and overarching dimensions to measure $\mathrm{MI}$ in $\mathrm{MH}$ for global use. An indicator provides a measure of a concept, and is typically used in quantitative research. Within this study, we focus on specific questions assessing $\mathrm{MI}$ in $\mathrm{MH}$ and consider each question as an indicator. ${ }^{30}$

\section{Literature review and initial list of indicators}

The initial list of indicators with their respective dimensions was developed by analysing and interpreting the results of a systematic review examining indicators used in the last 20 years to assess $\mathrm{MI}$ in $\mathrm{MH} .^{3}$ First, the most frequently used indicators were selected based on the review. Following that, the retrieved list of indicators was refined by examining the evidence base of each indicator, based on both quantitative and qualitative literature. Indicators reflecting MI that have been shown to contribute directly or indirectly to improved health outcomes for men, women or newborns were included. A final list (see online supplemental file 1; table 1) was compiled with background information regarding the use of the indicators. In the Delphi study, we worked with a survey questionnaire for men regarding their involvement in MH during the transition to parenthood. It is important 
to note that the survey was only a prototype to assess the indicators. Ideally, the survey should also be administered to women (see online supplemental file 2, table 1) and could be complemented with other data (such as reports from healthcare providers or other community members) for triangulation.

\section{Expert inclusion criteria, identification and recruitment}

A mixture of purposive sampling and systematic sampling was used to identify and recruit experts for the survey. Experts were recruited using three different strategies:

1. A search strategy was entered in Web Of Science to select papers regarding MI in MH. The top 20 authors (based on the number of peer-reviewed articles included in the search) were contacted to participate in the Delphi study.

2. Authors with two or more papers as first author included in the systematic review (unpublished data of the systematic review) were also included. This search strategy overlapped with the previous search strategy, resulting in 13 new authors who were approached for participation.

3. Finally, purposive sampling was conducted to include experts outside academia (mainly policymakers) by examining author lists of guidance documents on MI programmes and reviewing the speakers list of MI conferences and webinars. This was complemented by sending out emails to personal contacts within the field of $\mathrm{MH}$, requesting them to refer us to experts within the field of MI in MH. This strategy resulted in another 35 contacts.

Three additional experts were identified by snowball sampling, because some selected experts spontaneously referred us to their colleagues or personal contacts. In total, 71 people received an invitation email.

The online survey was developed with Sogosurvey ( www.sogosurvey.com). In October 2020, prior to data collection, the online survey was piloted by three senior researchers. Feedback from the pilot was incorporated in the final version of the first survey. Data were collected from October 2020 to December 2020.

The objective of this Delphi study was to present results based on the consensus of the group. ${ }^{31}$ An $85 \%$ agreement and no strong opposition in open text comments was defined as consensus. Maximum number of rounds was predefined at three rounds at the start of the study in order to inform potential participants about their required engagement beforehand. Participants could change their opinion based on other participants' input. If $85 \%$ of experts agreed to exclude an indicator, the indicator was dropped. Three rounds deemed enough to reach consensus. The original list of indicators consisted of 21 items and 6 domains (see online supplemental file 1: table 1), which was altered to 18 indicators and 5 domains after reaching consensus (see box 1).

\section{Patient and public involvement}

No patient or public involvement took place in the design or conduct of this study.

\section{First round: assessing weaknesses and strengths of each indicator}

All experts received the survey link by email together with detailed instructions and a video PowerPoint presentation explaining the development of the first round of the Delphi survey, including detailed information regarding the systematic review and selected indicators. Subsequently, experts were asked to rate the indicators on a Likert scale $(5=$ high relevance, $4=$ relevant, $3=$ moderate relevance, $2=$ low relevance and $1=$ not relevant) for validity, feasibility, sensitivity and specificity. For each category, experts were also asked to provide comments by open text. Validity referred to whether there is adequate evidence that this indicator reflects MI and that information of the indicator is useful for improving overall health outcomes. Feasibility referred to whether the required information could be collected easily from an existing source with limited missing values or other difficulties (such as social desirability or lack of complete medical records). Sensitivity referred to an indicator's ability to identify a partner/man who is actually involved as 'positive' by having also a positive (='yes') answer to that particular question. The specificity of an indicator referred to its ability to identify a man who is actually not involved as 'negative' by a negative response (='no') to the question. Experts received these definitions several times as reminders throughout the survey. They were also encouraged to recommend any other indicators that they deemed relevant but that were missing during the first round.

\section{Second round: retrieving the most valid and feasible indicators}

An average score for validity, feasibility, sensitivity and specificity scores (from the first round) was calculated for each indicator and a synthesis of the open text responses was made, which was shared with the experts in the second round. Furthermore, indicators were improved according to experts' feedback and new proposed indicators were added. Experts were asked to indicate whether they agreed or disagreed (being mutually exclusive) with the proposed indicators and to justify their decision in open text responses under each category.

\section{Third round: building consensus}

Experts received feedback regarding the previous round, with an explanation that only indicators with a minimum consensus of $85 \%$ agreement were included in the final framework, indicators below $85 \%$ were deleted or adapted based on the feedback. The threshold of $85 \%$ before inclusion was based on previously published standards within Delphi studies. ${ }^{32}$ Again, any adaptation or deletion of an indicator was explained by adding written information under the indicator. In this final round, 
experts were invited to make final open text comments on the selected indicators, including if they did not agree on certain retrieved indicators. Finally, they were also asked to propose which indicators were highly context specific (being mutually exclusive, Yes or No) according to their expertise. This question was added because of a general comment or concern of the experts in the previous rounds regarding the context specificity of certain indicators.

\section{Data analysis}

A Delphi study is often considered a mixed method approach with quantitative and qualitative data collection, and in this Delphi study both quantitative and qualitative data were triangulated to arrive at final results and conclusions. After three rounds, a high level of consensus was reached together with sufficient clarification of the different opinions and viewpoints. ${ }^{33} 34$

After the first round, the validity, feasibility, sensitivity and specificity of indicators were calculated by taking the mean of all the experts' scores in percentage. For easier interpretation of results by experts in the second round, scores of the first round were presented by reporting in which quintiles the indicator was situated compared with others. For exploring contrasting views, variability of the different scores was assessed by calculating the variance (see online supplemental file 3). After the second round, a consensus score (CS) was obtained, by calculating how many experts agreed to include the indicator in the list in percentage (eg, 100\% means all experts responded they would include the indicator). A scatterplot was used for visualising validity versus feasibility and sensitivity versus specificity. After the third round, a context specificity score (CSS) was calculated for each indicator, by calculating how many experts rated the indicator as 'highly context specific' in percentage (eg, 100\% means all experts responded that the indicator was highly context specific). CSs and CSSs were visualised by a lollipop plot (variation of a barplot ${ }^{35}$ ). CSs and context specificity ratings were retrieved in separate rounds, resulting in missing values in the visual for context specificity, because certain indicators were adapted, deleted or added after round 2. Missing values for CSSs (for indicator b3, fl and f2) were imputed by the mean.

Indicators were revised, taking into account all open text responses of experts (see later under 'qualitative data') and the rating scores. All statistical analyses and visualisations were conducted in Excel and R.

Open text comments were first analysed rapidly for feedback to the participants by looking at common themes inductively per indicator. For the purpose of this final paper, themes across all indicators were compared and synthesised into broader concepts, linking the comments back to the six broad categories deductively (for example, all concerns related to decision-making as a measurement of MI).
RESULTS

\section{Quantitative data}

Sociodemographic characteristics of participants

Respondents were purposively chosen to represent very different demographic backgrounds. Respondents' countries of origin included the Netherlands, Tanzania, Cameroon, Spain, USA (6), Canada (2), Philippines, Niger, Sweden (2), Japan, Poland, Norway, Mozambique (2), Belgium, Australia, Italy, Nigeria and Norway. Most respondents were doing academic work (23), some clinical work (3), some policy work (2), and some specified they were working in health systems strengthening (2). Some of them (4) combined more than one of these activities. Thirty-eight per cent of respondents (10) were male and 62\%(16) were female. Twelve respondents had a medical background (nine medical doctors, three midwives/nurses), two had a background in sociology, two in psychology and two in anthropology, and others (6) had a degree in biology, pharmacy, health economics or public health. Years of work experience ranged from 5 up to 42 years, with an average of 20 years.

\section{Response rates}

Thirty out of 71 email invitations were responded positively, of which 26 participants completed the first survey, resulting in a response rate of $37 \%$. Of those, 25 participants also completed the second survey and 24 the final survey. The completion rate of the Delphi study was $92 \%$ (number of respondents filling in the first survey divided by the number of respondents filling in all three surveys expressed as a percentage).

\section{Ratings}

During the first round, experts rated 23 indicators for validity, feasibility, sensitivity and specificity (see online supplemental file 1; table 1). In figure 1, scores for feasibility and validity are visualised. Rating scores ranged from $56 \%$ to $85 \%$ for validity and $64 \%$ to $84 \%$ for feasibility. Entering the ANC consultation (c2) and accompanying the partner to the facility for childbirth (c4) were considered to be particularly highly valid and feasible indicators. In figure 2, scores for sensitivity and specificity are visualised. Rating scores ranged from $67 \%$ to $87 \%$

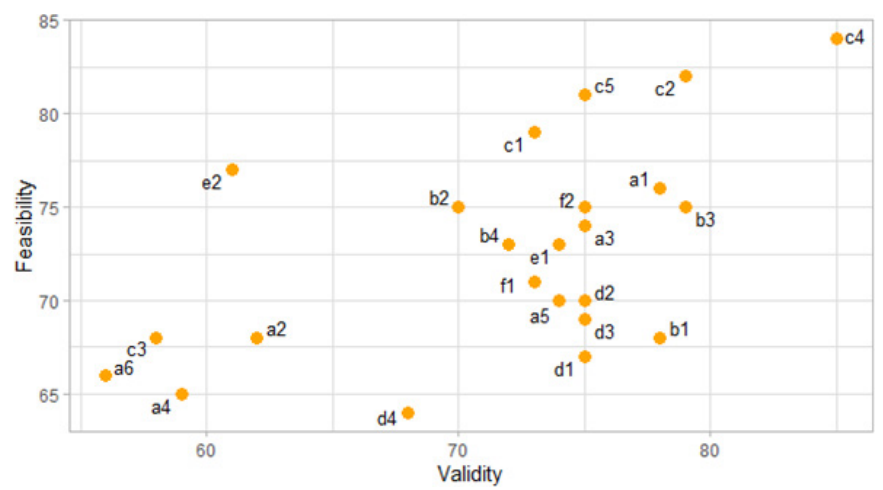

Figure 1 Scatterplot for validity and feasibility scores in percentage in round 1 of the Delphi study. 


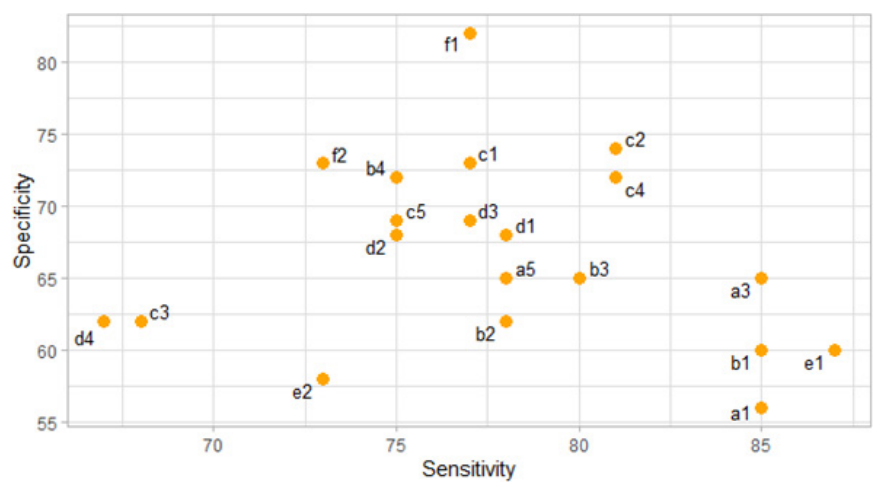

Figure 2 Scatterplot for sensitivity and specificity scores in percentage in round 1 of the Delphi study.

for sensitivity and $56 \%$ to $82 \%$ for specificity. The visual shows that some indicators were rated extremely high on sensitivity and low on specificity (eg, a1: Talking with the partner about ANC and e1: Acknowledging the child), while for other indicators, sensitivity and specificity scores seem to go hand in hand (eg, all indicators in the category 'physical involvement').

In round 1, respondents could also indicate which category name they preferred for the different categories (c) and (f). For category (f), 73\% preferred 'physical' over 'presential' and for category (c), $65 \%$ preferred 'cognitive' over 'intellectual'.

In round 2, several indicators were reworded, adapted and added, resulting in a list of 21 indicators (see online supplemental file 1; table 2). Experts were asked to indicate whether they agreed or disagreed with including the 21 proposed indicators, based on the received feedback from other participants. For three indicators that received low ratings and negative open text comments in the first round, experts were specifically asked if they agreed to exclude them from the list (see online supplemental file 1; table 2, c3 and e1). Those three indicators received high scores for exclusion (92\%, 88\%, 92\% of experts agreed to exclude) and as a consequence all three indicators were eliminated in the third round. Consensus scores for inclusion for all other indicators ranged from $56 \%$ to $100 \%$ (see figure 3). Consensus for inclusion was highest

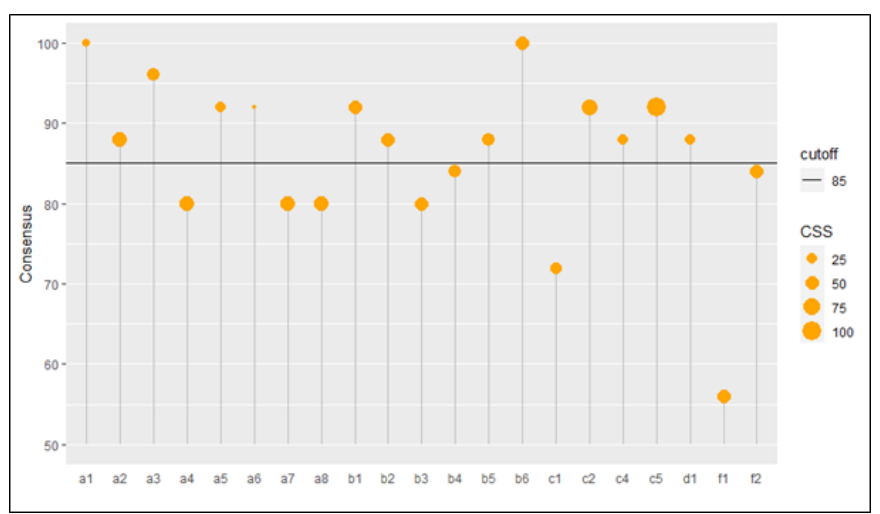

Figure 3 Consensus for the indicators of round 2 combined with context specificity scores (CSSs) of round 3 in percentage. for 'a1: Talking about ANC with the partner during pregnancy' and 'b6: Taking care of the baby' and lowest for ' $\mathrm{fl}$ : Knowing the content of ANC' and 'c1: Accompanying to the entrance of the facility for ANC'.

In round 3, a new list of 21 indicators was proposed, and experts rated them for context specificity, with scores ranging from $8 \%$ to $100 \%$. Two indicators from the category 'physical involvement' were rated as highly context specific, namely 'c2: Participation in ANC' and 'c5: Presence at childbirth'. In addition, they gave final input by open text comments (see further). Based on the final open text comments, the ultimate list of indicators was created (see box 1) with corresponding framework (see figure 4$)$.

\section{Qualitative data}

Involvement in communication and decision-making

Within the first category, indicators regarding communication were considered important with high scores on validity, feasibility and sensitivity. Specificity scores were lower, which was clarified by several experts in open text, explaining that 'did you communicate with' is a vague expression, open to misinterpretation. In some cases, couples might simply inform each other, while in others they might have an in-depth discussion, which would reflect varying levels of involvement. As noted by one of the respondents: 'One-way conversation or a direct order regarding attending or not attending antenatal care is not really communication although it could be interpreted as such.' No alternative indicator or 'solution' was proposed for resolving this concern, besides rewording 'communicate with' to 'talking with', and exploring the aspect of communication through qualitative data.

Another concern raised by experts was the unstandardised provision of PNC, which might affect the validity and feasibility of certain indicators (see online supplemental file 1 ; table 1 , a5 and a6). On the one hand, experts argued that PNC is not offered globally (although it is recommended by the $\mathrm{WHO}^{36}$ ) and as a consequence MI indicators should not focus on this care component along the maternal and newborn healthcare continuum. On the other hand, several experts reasoned that PNC is too often neglected, and argued that inclusion of an assessment of MI regarding this aspect of care is important as part of efforts to focus more attention on PNC in maternal healthcare programmes. Indicators regarding PNC were added based on the latter argument, but without focusing on male partner presence at the PNC. Based on the experts' comments and the absence of studies examining the potential benefits of involving partners in the PNC visit for women, it was decided male presence at PNC cannot be considered as an evidencebased MI indicator at this time.

Experts had mixed opinions regarding the indicators about 'decision-making', with some supporting 'shared decision-making' as an indicator of MI, while others believed women should always be encouraged to take decisions autonomously. Consequently, some experts 
Box 1 Final list of indicators for assessing male involvement in maternal health-male version

1. Involvement in communication

a. Did you talk with your partner about what happens during the antenatal care consultations (=care during pregnancy at the healthcare centre)?

b. During the pregnancy, did you talk with your partner about the place of birth?

c. Did you talk with your partner about what happens during the postnatal care visit for women (a visit to the health centre after giving birth for checking the mother)?

d. Did you talk with your partner about what happens during the first newborn care visit (a visit to the health centre after giving birth for checking the baby)?

2. Involvement in decision-making

a. Did you take part in the decision whether or not to go for antenatal care?

b. Did you take part in the decision about the place of birth?

c. Did you take part in the decision whether or not to go to the postnatal care visit (for checking the woman after childbirth)?

d. Did you take part in the decision whether or not to go for the first newborn care visit at the healthcare centre?

3. Practical involvement

a. Did you provide any type of financial support (including transportation) during the pregnancy (for reaching the health facility, buying medication)?

b. Did you participate in doing household chores (such as washing clothes, cleaning the house, washing dishes, preparing meals) during the pregnancy?

c. Did you keep money aside during pregnancy for childbirthrelated expenses (such as baby clothes, medical fees, transport)?

d. Did you plan transportation to the nearest health facility in case of complications or emergencies during the pregnancy or period after birth?

e. Did you participate in taking care of the baby (for example, giving a bath, changing diapers, carrying the baby) in the first weeks after delivery?

4. Physical involvement

a. Did you accompany your partner to the health facility for antenatal care?

b. Did you participate in the antenatal care consultation (=entering and receiving the information/care)?

c. Did you accompany your partner to the health facility for giving birth?

d. Where you present during labour or childbirth as a birth companion?

e. Did you participate in the first newborn care visit (first examination of the newborn at the facility, done within 6 weeks after childbirth)?

5. Emotional involvement

a. Overall, I feel I supported my partner emotionally during the pregnancy.

b. Overall, I feel I supported my partner emotionally during labour and childbirth.

c. Overall, I feel I supported my partner emotionally in the first weeks after childbirth.

believed that 'shared decision-making' or a male partner making the decision might reflect male dominance rather than MI. Opinions varied regarding inclusion of
Decision

making

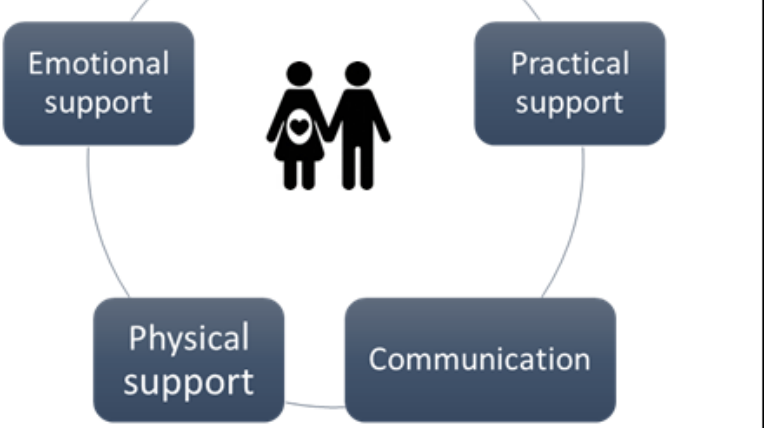

Figure 4 Global framework for assessing male involvement in maternal health.

decision-making as an indicator and how to frame it. An alternative question of "whether the man participated in the decision' was proposed in the open text comments, which was further explored in the second round and generated more consensus. Because of the concerns regarding certain indicators assessing male dominance instead of MI, we added a note recommending collection of additional data regarding decision-making (qualitative data) and gender equality (eg, Promundo indicators ${ }^{37} 38$ ) when using the MI framework in future research. This information was added to the final round and experts agreed with this approach. Another concern regarding the validity of the decision-making questions in resourcelimited settings was that when institutional healthcare unavailable or very limited, these indicators might not reflect MI. As expressed by one participant 'this may not be much of a decision for many families'. To reflect this, we added a note to the final list of indicators to recommend collection of additional data regarding sociodemographic characteristics of respondents to explore factors such as poverty, distance to the health facility and workrelated barriers (such as partners working abroad or with strict working hours).

Lastly, the open text comments in the final round revealed that several experts considered this category as reflecting two distinct issues: communication and decision-making. Therefore, this category was divided into two separate categories in the final framework, one about communication and one about decision-making (see figure 4).

\section{Practical involvement}

Overall, indicators within this category were evaluated positively, and were considered clear and straightforward, although some general concerns were raised. A general comment from most respondents was the high risk of social desirability bias within this category, especially in settings where a financial contribution is expected from the male partner. This was expressed as follows: 'Social desirability bias may have a substantial impact on results. 
This type of support typically aligns with men's established gender role (eg, controlling household resources).' Unfortunately, social desirability could be an issue for the majority of the indicators and it is not possible to completely avoid this kind of bias. Nevertheless, since the list of indicators is designed to be used for data collection among both men and women, this could help in revealing contradicting findings related to social desirability.

The other main experts' comments related to context specificity, which will need to be addressed in the future, by slightly adapting the questions to the context and available resources (such as commonly available transport, the financial contribution that is expected during pregnancy/childbirth and health system factors). As recommended by the experts, an indicator regarding participation of men in taking care of the baby was added (see online supplemental file 1; table 2, b6), with high agreement among experts in the second round.

\section{Physical involvement}

In this category also, context specificity was a concern among several experts. It was felt that the indicators might not capture MI, especially in contexts where men are not allowed to be present during certain elements of maternity care (ANC, childbirth or PNC) or when cultural norms prohibit men from being present. This was explained as follows: "Although I agreed with those indicators, it will be hard in most African cultures to have a man as birth companion.'

One specific indicator, HIV testing, received low scores on all domains (validity, feasibility, sensitivity and specificity) but also with high variance for validity, indicating low consensus (see online supplemental file 3). Several experts argued this indicator is a reflection of the quality of ANC or Prevention of Mother-To-Child Transmission (PMTCT) programme success rather than MI: 'To me this indicates the health system (ie, is couples voluntary counselling and testing integrated with ANC) rather than men's involvement. In high-HIV prevalence settings, HIV counselling cannot really be measured separately from attendance at the first ANC visit (or at least one ANC visit).' The majority of experts argued that the indicator does not distinguish involved from non-involved men. This was illustrated by the following comment: 'A man may already know he has HIV and therefore may not get counselling. But this wouldn't suggest his attitude about the pregnancy.' Based on the comments and ratings, HIV testing was deleted as indicator within the list.

Because of previous research indicating that men, women, health providers and researchers themselves interpret male accompaniment at ANC differently (accompanying to the health centre vs actually entering the consultation room), we tried to capture this difference by developing two separate indicators for male ANC attendance (see online supplemental file 1; table 1; c1: Did you accompany your wife to the entrance of the health facility for ANC? and c2: Did you enter the ANC consultation room?). Experts agreed that both aspects are important and constitute involvement, but criticised the wording, in particular the term 'entrance' was found to be confusing. The two indicators were reworded and improved throughout the rounds of the Delphi process to express the difference more clearly, based on the experts' input. In the final round, no further concerns were raised.

The overall category was considered to be an essential part of the framework by a majority of the experts, as expressed by one participant in the last round: 'I consider all items as very relevant measuring male involvement whether in the rural periphery in low income countries or urban, or in the North. It is difficult to state the items are context specific in the rapid changing landscape of facility delivery attendance worldwide. There is a necessity for such an instrument to measure changes in behaviour over time in different contexts.'

\section{Emotional involvement}

For many experts, this aspect of MI was seen as progressive and important, although they felt the indicators might be hard to be interpreted or understood, especially in contexts where psychosocial assessments are rarely carried out. Based on the comments, there was consensus regarding the importance of including a question about the subjective feeling of support, although the translation into the four proposed indicators was not unanimously appreciated. Some experts argued that capturing deeper emotional aspects (such as sharing feelings and joy) are not core elements of an MI list of indicators and are too culture specific. One expert commented: 'In my opinion this measures emotional intimacy and emotional support within a couple (or coparent) relationship, which is closely related to, but distinct from, men's involvement.' The replacement of the four initial indicators (see online supplemental file 1 ; table $1, \mathrm{~d} 1-\mathrm{d} 4$ ) by more general questions (see figure 4, category Emotional support) was positively evaluated and considered an improvement by most experts. The inclusion of other periods (childbirth and post partum) within this category was suggested by the participants to align with the objective of the framework (which is to capture MI during pregnancy, childbirth and post partum). Nevertheless, some experts perceived that the absence of indicators assessing mutual support (including support of the woman towards the male partner) and wider emotional aspects of support was a shortcoming of the final set of simplified indicators.

\section{Legal involvement}

Scores regarding legal involvement were rather low on all domains for both indicators but also with a high variance for validity and feasibility (see online supplemental file $3)$. From the open text comments, it was clear that at least half of the respondents did not consider this to be an essential MI indicator, especially because the same men that recognise the child might not be involved (resulting in low specificity scores). Nevertheless, some experts also highlighted that legal acknowledgement affects women's sense of security and role in society, making it an 
important indicator. An expert raised the following argument for inclusion of the acknowledgement indicators: 'Voluntary establishment of legal paternity can be a proxy for both paternity confidence and male commitment to investing in the children, all of which reduces the anxiety of pregnant women and potentially improves maternal health outcomes.' However, a majority of the experts agreed that child acknowledgement and having the father's name on the birth certificate are not core indicators of MI, but that these variables are important to track in MI research, in similarity with other sociodemographic characteristics such as marital status and poverty level. In conclusion, acknowledgement of the child was concluded to be an inadequate measure of paternal investment and therefore deleted from the list.

\section{Cognitive involvement}

While a majority of experts found these indicators to be important, with limited variance among respondents (see online supplemental file 3), several concerns were raised in open text responses. First, several experts agreed that male partners' knowledge of danger signs might depend on their ability to recall items, as well as literacy and access to information (eg, information provided during ANC), rather than actual 'involvement'. One expert highlighted the following: 'It depends on their levels of understanding as well as literacy and commitment of health care providers. So I felt that these questions can measure only a small part of the involvement.' Furthermore, the calculation of what was considered as 'good knowledge' for men, and as a consequence also 'involved', was seen as invalid and oversimplified. This was expressed as follows: 'This is very didactic. What knowledge is most relevant and useful in a male partner?'

In conclusion, it seemed that experts agreed that the assessment of knowledge of danger signs was important, but was not a reflection of being involved or not. In the final round of the Delphi survey, several experts indicated that, after reviewing the complete framework, they considered the last category as non-essential. One respondent explained this as follows: 'I don't see what these questions will add besides what is already known from the previous indicators.' Therefore, this category was eventually deleted, despite the importance attributed to knowledge of danger signs by many experts within MI research.

\section{DISCUSSION}

From consulting the literature and based on our own research experience, we noticed a knowledge gap within $\mathrm{MH}$ care research regarding valid and feasible indicators for assessing MI in MH. As a first step, a systematic review was conducted regarding the conceptualisation of MI in $\mathrm{MH}$ and most commonly used indicators. ${ }^{3}$ The retrieved list of indicators from the systematic review then required a critical review from a global perspective for practical use in the future. Therefore, a Delphi study, involving experts from all continents, was the next step towards our goal of presenting a global framework for assessing MI in MH. Key components of a Delphi process were followed, including anonymity, iteration, controlled acquisition of feedback and analytical aggregation of responses. ${ }^{34}$ Experts were encouraged to reflect on a global MI framework, outside the scope of their own research activities to minimise bias. Round-to-round dropout rates were extremely low, which could be related to the targeted selection of participants (based on expertise and interest in the topic) and use of personal emails as reminders. The outcome of our study is a global MI framework with a list of indicators, open for improvements and adaptations. The main implications of the findings of this Delphi study will be discussed, taking into account the following aspects: (1) contrasting and unanimous views of experts, (2) practical use of the framework, (3) strengths and limitations, and (4) further research.

Our initially proposed list of 23 evidence-based indicators was multidimensional (including six different dimensions or categories) and based on the most commonly used indicators within the literature from the last 20 years. Experts agreed on the importance and inclusion of most of the indicators within the dimensions, although almost all of the indicators were slightly adapted or reworded according to experts' input. The most contrasting viewpoints among experts were directed at the categories relating to decision-making, acknowledgement of the child and knowledge of danger signs as proxies for MI.

The first category, decision-making, included questions regarding communication and shared decision-making. Most research regarding the importance of communication and shared decision-making within an assessment of MI derives from family planning studies. Several researchers have shown that improved communication about reproductive health can lead to shared decision-making, which can in turn lead to improved access to family planning services. ${ }^{39}{ }^{40}$ Redshaw and Henderson ${ }^{41}$ were one of the first researchers to include decision-making during pregnancy within an MI assessment in 2013, while Ampt $e t a l^{24}$ were quite progressive by including both communication and decision-making as essential elements of their index to measure MI in maternal and newborn health in 2015. Both communication and shared decision-making as MI measurements became much more common from 2015 onwards within the literature. ${ }^{42-44}$ Today, the importance of assessing communication within MI research seems to be generally recognised, and there was also high agreement among experts in our study for the inclusion of indicators related to communication. The main drawback of using communication to assess involvement, noted by respondents within our study and other researchers, ${ }^{24}$ is the risk of assessing male dominance rather than involvement. In fact, this argument is also applicable to several other quantitative MI indicators. Triangulation of data (including in-depth qualitative research and reports from different sources such as men, women and health providers) could potentially distinguish MI from male dominance. In relation to the specific aspect of 
decision-making, much less consensus exists regarding what is desirable within MH (shared decision-making vs women making autonomous decisions), both in our study and in the literature. While certain more feminist groups have aimed to empower women to make their own decisions about reproductive health within $\mathrm{MH}$ care programmes, ${ }^{45-47}$ other stakeholders emphasise that communication and shared decision-making with the partner leads to healthier relationships and better health outcomes. ${ }^{39} 4849$ The literature also reflects this troubled relationship between female empowerment and MI, with contradicting findings on whether the two concepts are positively correlated or not. ${ }^{50}$ In our list of indicators, we included questions regarding the participation of men in decision-making, a measurement whereby supporting a woman in her decision can also be considered as participation and constitutes positive MI. Nevertheless, the decision-making category is probably the most debatable part of the framework, leaving room for further improvements and follow-up research.

Another category in our framework, acknowledgement of the child, also received divergent opinions from experts in the first round, although a final consensus regarding omitting the indicators was obtained by the end of the study. Acknowledgement of the child, or a proxy such as having the father's name on the birth files, has been used to assess the relationship between MI and neonatal health outcomes in several large cohort studies. ${ }^{52-54}$ Notably, those studies often relied retrospectively on large national or international datasets, whereby a more nuanced assessment of MI was not possible. Based on the experts' feedback, it became clear that whether or not a child is recognised by the male partner is an inadequate measurement of MI and adds little extra information to the other indicators of the list. In order to align with our goal of only including the most valid indicators, this category was eventually deleted from the framework.

The last category with conflicting experts' opinions concerned knowledge of ANC content and danger signs as measures of MI. The most important criticism was that the male partner's knowledge will depend mainly on the quality of ANC and his ability to recall items, rather than his actual involvement. Furthermore, knowledge of danger signs is often low among men and women in general, ${ }^{55-57}$ suggesting that this has little to do with being involved or not. However, some experts considered knowledge of danger signs to be an essential aspect of MI, emphasising that it may have a direct impact on MH outcomes. Within the literature, we also observed different constructs: on the one hand a partner with good knowledge of danger signs was considered as being involved ${ }^{58}$ while on the other hand the knowledge of danger signs and MI were seen as two different concepts, but often with a positive correlation. ${ }^{575960}$ While several studies have demonstrated the importance of knowledge of danger signs among women for complication preparedness, ${ }^{6162}$ the evidence regarding the added value of men's knowledge of danger signs in improving health outcomes, as well as what can be defined as 'good knowledge' for a male partner, is less clear. The category 'knowledge of content of ANC and danger signs' was eventually deleted from the framework because of the lack of consensus both among the experts and within the literature, although it is an important aspect within $\mathrm{MH}$ that requires further research.

Our final MI framework and corresponding list of indicators are intended to be used for an assessment of men's involvement during pregnancy, childbirth and the postpartum period. All indicators were carefully selected based on experts' input and the evidence base, meaning that only aspects of MI that have been shown to contribute to improved health outcomes were included. While we incorporated indicators related to partners' presence during ANC and childbirth, we did not include a similar indicator for assessing their presence during the PNC. Experts agreed to this approach within our study, because the added value for inviting men during PNC is not yet demonstrated in the literature, neither is it recommended by the WHO. ${ }^{4} 366364$ On the other hand, indicators related to men's practical support and emotional support post partum within the household were included, because those aspects of MI have shown to be beneficial for maternal and newborn health outcomes, especially for preventing postpartum depression. ${ }^{45}$ Nevertheless, $\mathrm{PNC}$ is organised differently in certain settings. One PNC visit can include family planning counselling, weighing of the newborn and a medical check-up of the woman as integrative approach (as recommended by $\mathrm{WHO}^{66}$ ) or care might be fragmented in different visits (eg, women are seen by a gynaecologist and newborns by a neonatologist) ${ }^{67}$ As a consequence, the proposed indicators might need to be specified according to different contexts. Overall, we found a gap in the literature regarding male participation in PNC, which might need further investigation in the future.

The final indicators are presented here as a survey for the male partner, however it is highly recommended that MI studies also collect data from women, given the often contradicting findings between men and women ${ }^{57}$ which require further investigation. A female version of the list of indicators was added in online supplemental file 2 to facilitate data collection among both men and women. Furthermore, other sources such as reports from healthcare providers and medical registry data could be used to complement the indicators together with sociodemographic data for correct interpretation. Poverty, distance to the health facility and work-related restrictions (such as partners working abroad or with strict working hours) are all factors that might prevent men from being involved during pregnancy and childbirth, which can only be explored by collecting the necessary quantitative and qualitative data in addition to the list of indicators. Ideally, the MI list of indicators and corresponding framework is used for data collection in the postpartum period, although also administration during other time periods is possible, with consideration of recall bias and/or adaptations of the questions. Furthermore, we recommend that 
scores are presented per category, rather than an overall 'MI score', because the latter loses nuances and could be challenging to interpret. Finally, the experts' assessment of context specificity of the indicators taught us that while some indicators are likely to be very useful globally, others might need adaptations. We strongly recommend that researchers who intend to use the list of indicators and corresponding framework review each indicator, especially those with a high score on context specificity, and adapt them to the context, taking into account health system factors as well as cultural and social norms.

\section{Limitations}

The development of a global framework to measure MI in $\mathrm{MH}$ is quite novel and several aspects of this topic are still understudied within the literature. Consequently, the framework should be considered as a flexible tool rather than a rigid instrument, that might take different forms according to the context and newly available evidence. Another limitation is the rather short and intense time span in which this Delphi study was conducted, which was chosen in order to guarantee a high response rate. While the high response rate is a strength of this study, the short time span did not allow a long reflection time for respondents, and also did not permit a real-time virtual or in-person meeting with the experts. The latter could offer more exchange of ideas and more nuanced views on the framework, but is also under debate within Delphi studies because of a lack of anonymity. In the aftermath of the COVID-19 pandemic, where many research activities were replaced by an online format, an evaluation study could explore the strengths and weaknesses of Delphi studies being conducted entirely online. Lastly, pregnant women and their partners were not involved in the development of the framework, which is a limitation. In light of the mentioned shortcomings, we would like to emphasise that the framework is open for improvements and adaptations, based on new insights and evidence in the future.

\section{Further research}

To our knowledge, this is the first study proposing a global framework for MI in MH. The MI framework could be a first step towards more standardised evaluations of MI programmes and may allow easier interpretation of evidence in different contexts. Nevertheless, the framework and list of indicators need to be piloted and validated in different countries, together with an evaluation of the strengths and weaknesses of using the proposed indicators compared with previously used MI assessments. Furthermore, men's role in family planning decision-making and uptake was not explored as a part of the framework, neither was men's role in access to abortion care. While both domains can be viewed as essential parts of maternal healthcare, they were considered to be beyond the scope of this study and would have required a broader and more in-depth analysis of the available evidence. The assessment of MI in these specific aspects of $\mathrm{MH}$ could be explored in follow-up research. Pilot studies for evaluating and validating the framework are planned within our research group, but we also encourage other researchers to use, validate and disseminate this framework widely and to contact us for collaborations.

\section{CONCLUSION}

This study explored the opinions of experts from diverse backgrounds and regions on the validity and feasibility of a list of key indicators for measuring MI in MH globally, by using the Delphi method. A global multidimensional MI framework was constructed, based on the expert panel input and evidence from the literature. While we aimed for consensus in the indicator selection process, we also documented different views and perceptions among experts and within the literature. The proposed list of MI indicators and corresponding framework intend to capture the concept of MI in MH at local, national, and international levels, which we believe could allow improved assessment and comparison of study findings, eventually leading to better programming and health outcomes in $\mathrm{MH}$.

Acknowledgements We would like to thank all the experts who were willing to take part in this Delphi study for their valuable contributions. Their suggestions, based on numerous years of experience with the topic, gave us the opportunity to pursue our objective of constructing a global male involvement framework. Furthermore, we would like to thank the colleagues from ICRH-Belgium for their continuous support and encouragement during the study.

Contributors $A G$ and $O D$ conceptualised the study and $A G$ led data collection and analysis. KR and NO gave input on the initial list of indicators and SG and OD contributed to the development of the online survey. Interpretation of the data was done by AG with contributions from OD, SG, NO and KR. AG drafted the final manuscript and OD, SG, KR and NO gave input during writing. All authors read and approved the final version of the manuscript.

Funding AG is funded by a VLADOC PhD scholarship from the Flemish InterUniversity Council (VLIR-UOS Belgium).

Disclaimer The funder had no role in the study design, data collection, analysis, interpretation of data or in writing the manuscript. Researchers are independent from funders and all authors had full access to all data (including statistical reports and tables) in the study and can take responsibility for the integrity of the data and the accuracy of the data analysis.

Competing interests None declared.

Patient consent for publication Not required.

Ethics approval All participants were invited by email 1 month before the start of the study and interested participants were asked to respond positively by email. Each online survey started with an informed consent statement whereby participants had to indicate if they had understood the information and agreed to participate. The identity of each member was anonymous to other members of the panel and was known only to the principal investigator (AG). Ethical approval for this study was obtained from the Bioethics Committee of Ghent University (EC/2018/1319)

Provenance and peer review Not commissioned; externally peer reviewed.

Data availability statement Data are available upon reasonable request. Data analysed during the current study will be made available from the corresponding author upon reasonable request.

Supplemental material This content has been supplied by the author(s). It has not been vetted by BMJ Publishing Group Limited (BMJ) and may not have been peer-reviewed. Any opinions or recommendations discussed are solely those of the author(s) and are not endorsed by BMJ. BMJ disclaims all liability and responsibility arising from any reliance placed on the content. Where the content includes any translated material, BMJ does not warrant the accuracy and reliability of the translations (including but not limited to local regulations, clinical guidelines, 
terminology, drug names and drug dosages), and is not responsible for any error and/or omissions arising from translation and adaptation or otherwise.

Open access This is an open access article distributed in accordance with the Creative Commons Attribution Non Commercial (CC BY-NC 4.0) license, which permits others to distribute, remix, adapt, build upon this work non-commercially, and license their derivative works on different terms, provided the original work is properly cited, appropriate credit is given, any changes made indicated, and the use is non-commercial. See: http://creativecommons.org/licenses/by-nc/4.0/.

ORCID iD

Anna Galle http://orcid.org/0000-0003-1427-5067

\section{REFERENCES}

1 EuroPROFEM. Male involvement in reproductive health: ICPD-Family care international, 2020. Available: http://www.europrofem.org/contri/ 204 en/en-masc/53en mas.htm

2 Wentzell EA, Inhorn MC. Reconceiving masculinity and 'men as partners' for ICPD Beyond 2014: insights from a Mexican HPV study. Glob Public Health 2014;9:691-705.

3 Galle A, Plaieser G, Van Steenstraeten T, et al. Systematic review of the concept 'male involvement in maternal health' by natural language processing and descriptive analysis. BMJ Glob Health 2021;6:e004909.

4 Yargawa J, Leonardi-Bee J. Male involvement and maternal health outcomes: systematic review and meta-analysis. J Epidemiol Community Health 2015;69:604-12.

5 Tokhi M, Comrie-Thomson L, Davis J, et al. Involving men to improve maternal and newborn health: a systematic review of the effectiveness of interventions. PLoS One 2018;13:e0191620.

6 Tadesse K, Zelenko O, Mulugeta A, et al. Effectiveness of breastfeeding interventions delivered to fathers in low- and middle-income countries: a systematic review. Matern Child Nutr 2018;14:e12612.

$7 \mathrm{MC} \mathrm{L}$, Jones L, Bond $\mathrm{MJ}$, et al. Where is the $\mathrm{F}$ in $\mathrm{MCH}$ ? father involvement in African American families. Ethnicity and Disease 2010;20 1 SUPPL.2:S249-61.

8 Tokhi M, Comrie-Thomson L, Davis J, et al. Involving men to improve maternal and newborn health: a systematic review of the effectiveness of interventions. PLoS One 2018;13:e0191620.

9 Triulzi I, Palla I, Ciccacci F, et al. The effectiveness of interventions to involve men living with HIV positive pregnant women in low-income countries: a systematic review of the literature. BMC Health Serv Res 2019;19:943.

10 Galle A, Griffin Icrh-M S, Nafissa M. A systematic review of the concept "male involvement in maternal health" by natural language processing and descriptive analysis. medRxiv 2021.

11 Byamugisha R, Tumwine JK, Semiyaga N, et al. Determinants of male involvement in the prevention of mother-to-child transmission of HIV programme in eastern Uganda: a cross-sectional survey. Reprod Health 2010;7:12.

12 Yende N, Van Rie A, West NS, et al. Acceptability and preferences among men and women for male involvement in antenatal care. $J$ Pregnancy 2017;2017:1-8.

13 Nyondo-Mipando AL, Chimwaza AF, Muula AS. A descriptive study on demographic and behavioral characteristics of males and their responses to a male involvement intervention in Blantyre, Malawi. Pan African Medical Journal 2016;25:229.

14 Berti PR, Sohani S, Costa Eda, da CE, et al. An adequacy evaluation of a maternal health intervention in rural Honduras: the impact of engagement of men and empowerment of women. Rev Panam Salud Publica 2015;37:90-7.

15 Lee JY, Knauer HA, Lee SJ, et al. Father-Inclusive perinatal parent education programs: a systematic review. Pediatrics 2018;142. doi:10.1542/peds.2018-0437. [Epub ahead of print: 1406 2018]

16 Montgomery E, van der Straten A, Torjesen K. "Male involvement" in women and children's HIV prevention: challenges in definition and interpretation. J Acquir Immune Defic Syndr 2011;57:e114-6.

17 Alaofè H, Zhu M, Burney J, et al. Association between women's empowerment and maternal and child nutrition in Kalalé district of northern Benin. Food Nutr Bull 2017;38:302-18.

18 Oyugi E, Gura Z, Boru W, et al. Male partner involvement in efforts to eliminate mother-to-child transmission of HIV in Kisumu County, Western Kenya, 2015. Pan Afr Med J 2017;28:6.

19 Galle A, Manaharlal H, Griffin S, et al. A qualitative study on midwives' identity and perspectives on the occurrence of disrespect and abuse in Maputo city. BMC Pregnancy Childbirth 2020;20.
20 Maman S, Moodley D, Groves AK. Defining male support during and after pregnancy from the perspective of HIV-positive and HIV-negative women in Durban, South Africa. J Midwifery Womens Health 2011;56:325-31.

21 Greenhill E, Vollmer RL. Perceptions of a Father's Role during a Couple's First Pregnancy. Family and Consumer Sciences Research Journal 2019;48:52-64.

22 Davis J, Vyankandondera J, Luchters S, et al. Male involvement in reproductive, maternal and child health: a qualitative study of policymaker and practitioner perspectives in the Pacific. Reprod Health 2016;13:81.

23 Mwendwa P, Karani C, Kamolo E, et al. What contextual factors and mechanisms facilitate male involvement in women's sexual and reproductive health in sub-Saharan Africa? a rapid realist review protocol. HRB Open Res 2020;3:55.

24 Ampt F, Mon MM, Than KK, et al. Correlates of male involvement in maternal and newborn health: a cross-sectional study of men in a peri-urban region of Myanmar. BMC Pregnancy Childbirth 2015; $15: 122$

25 Byamugisha R, Åstrøm AN, Ndeezi G, et al. Male partner antenatal attendance and HIV testing in eastern Uganda: a randomized facilitybased intervention trial. J Int AIDS Soc 2011;14:43.

26 Hampanda K, Abuogi L, Musoke P, et al. Development of a novel scale to measure male partner involvement in the prevention of mother-to-child transmission of HIV in Kenya. AIDS Behav 2020;24:291-303.

27 Hasson F, Keeney S, McKenna H. Research guidelines for the Delphi survey technique. J Adv Nurs 2000;32:1008-15.

28 Zhong S, Clark M, Hou X-Y, et al. Development of key indicators of hospital resilience: a modified Delphi study. J Health Serv Res Policy 2015;20:74-82.

29 Nair R, Aggarwal R. Rheumatism DK-S in arthritis and, 2011 undefined. Methods of formal consensus in classification/ diagnostic criteria and guideline development. Amsterdam: Elsevier, 2011

30 Jupp V. The SAGE dictionary of social research methods. Thousand Oaks: sage, 2011.

31 Diamond IR, Grant RC, Feldman BM, et al. Defining consensus: a systematic review recommends methodologic criteria for reporting of Delphi studies. J Clin Epidemiol 2014;67:401-9.

32 Keeney S, Hasson F, Mckenna H. The Delphi technique in nursing and health research. Hoboken: John Wiley \& Sons, 2010.

33 Rauch Wolf. The decision Delphi. Technol Forecast Soc Change 1979;15:159-69 https://www.sciencedirect.com/science/article/abs/ pii/0040162579900118

34 von der Gracht HA. Consensus measurement in Delphi studies. review and implications for future quality assurance. Technological Forecasting and Social Change 2012;79:1525-36.

35 The R Graph Gallery. Lollipop plot, 2021. Available: https://www.rgraph-gallery.com/lollipop-plot.html

36 WHO. WHO recommendations on postnatal care of the mother and newborn. Geneva: WHO, 2020.

37 Promundo - EN. International men and gender equality survey, 2021 Available: https://promundoglobal.org/programs/international-menand-gender-equality-survey-images/

38 Doyle K, Levtov RG, Barker G, et al. Gender-transformative Bandebereho couples' intervention to promote male engagement in reproductive and maternal health and violence prevention in Rwanda: Findings from a randomized controlled trial. PLoS One 2018;13:e0192756.

39 Hartmann M, Gilles K, Shattuck D, et al. Changes in couples' communication as a result of a male-involvement family planning intervention. J Health Commun 2012;17:802-19.

40 Mboane R, Bhatta MP. Influence of a husband's healthcare decision making role on a woman's intention to use contraceptives among Mozambican women. Reprod Health 2015;12:36

41 Redshaw M, Henderson J. Fathers' engagement in pregnancy and childbirth: evidence from a national survey. BMC Pregnancy Childbirth 2013;13:70.

42 Vermeulen E, Solnes Miltenburg A, Barras J, et al. Opportunities for male involvement during pregnancy in Magu district, rural Tanzania. BMC Pregnancy Childbirth 2016:16:66.

43 Gibore NS, Bali TAL, Kibusi SM. Factors influencing men's involvement in antenatal care services: a cross-sectional study in a low resource setting, central Tanzania. Reprod Health 2019;16:52

44 Lechowicz ME, Jiang Y, Tully LA, et al. Enhancing father engagement in parenting programs: translating research into practice recommendations. Aust Psychol 2019;54:83-9.

45 Abrar-ul-haq M, Jali MRM, Islam GMN. Decision-Making ability as a source of Empowerment among rural women of Pakistan. Global Social Welfare 2017:4:117-25. 
46 Prata N, Tavrow P, Upadhyay U. Women's empowerment related to pregnancy and childbirth: introduction to special issue. $B M C$ Pregnancy Childbirth 2017;17:352.

47 . Women's empowerment and gender equality: essential goals for saving women's lives. Geneva World Health Organization; 2008. https://www.who.int/gender/documents/EN_womens_emp.pdf [Accessed 10/10/2020].

48 Doyle K, Kato-Wallace J. Male engagement in maternal, newborn, and child Health/Sexual reproductive health and rights 2016.

49 Doyle K, Levtov RG, Barker G, et al. Gender-transformative Bandebereho couples' intervention to promote male engagement in reproductive and maternal health and violence prevention in Rwanda: findings from a randomized controlled trial. PLoS One 2018;13:e0192756.

50 Jennings L, Na M, Cherewick M, et al. Women's empowerment and male involvement in antenatal care: analyses of demographic and health surveys (DHS) in selected African countries. BMC Pregnancy Childbirth 2014;14:297.

51 Mullany BC, Hindin MJ, Becker S. Can women's autonomy impede male involvement in pregnancy health in Katmandu, Nepal? Soc Sci Med 2005;61:1993-2006.

52 Knight B, Shields B, Powell R. Paternal details missing at Booking: an identifiable risk factor for lower birthweight. Evidence Based Midwifery 2006;4:41-5.

53 Alio AP, Kornosky JL, Mbah AK, et al. The impact of paternal involvement on feto-infant morbidity among whites, blacks and Hispanics. Matern Child Health J 2010;14:735-41.

54 Merklinger-Gruchala A, Jasienska G, Kapiszewska M. Paternal investment and low birth weight - The mediating role of parity. PLoS One 2019;14:e0210715.

55 August F, Pembe AB, Mpembeni R, et al. Men's knowledge of obstetric danger signs, birth preparedness and complication readiness in rural Tanzania. PLoS One 2015;10:e0125978.

56 Mwilike B, Nalwadda G, Kagawa M, et al. Knowledge of danger signs during pregnancy and subsequent healthcare seeking actions among women in urban Tanzania: a cross-sectional study. BMC Pregnancy Childbirth 2018;18:4.
57 Galle A, De Melo M, Griffin S, et al. A cross-sectional study of the role of men and the knowledge of danger signs during pregnancy in southern Mozambique. BMC Pregnancy Childbirth 2020;20:572.

58 Assaf S, Davis LM. DHS analytical studies no. 64 does men's involvement improve the health outcomes of their partners and children? Rockville, Maryland, USA: ICF, 2018.

59 Nwakwuo GC, Oshonwoh FE. Assessment of the level of male involvement in safe motherhood in southern Nigeria. J Community Health 2013;38:349-56.

60 Worku M, Boru B, Amano A, et al. Male involvement and associated factors in birth preparedness and complication readiness in Debre Berhan town, North East Ethiopia. Pan Afr Med J 2020;35:36.

61 Mbalinda SN, Nakimuli A, Kakaire O, et al. Does knowledge of danger signs of pregnancy predict birth preparedness? A critique of the evidence from women admitted with pregnancy complications. Health Res Policy Syst 2014;12:60.

62 Jungari S. Informed motherhood: Women's knowledge of danger signs of obstetric complications and birth preparedness in low income communities in India. Child Youth Serv Rev 2020;117:105276.

63 Carter MW, Speizer I. Salvadoran fathers' attendance at prenatal care, delivery, and postpartum care. Revista Panamericana de Salud Pública 2005;18:149-56.

64 Sacks E, Langlois Étienne V. Postnatal care: increasing coverage, equity, and quality. Lancet Glob Health 2016;4:e442-3.

65 Gausia K, Fisher C, Ali M, et al. Magnitude and contributory factors of postnatal depression: a community-based cohort study from a rural subdistrict of Bangladesh. Psychol Med 2009;39: 999-1007.

66 Organization WH. Postnatal care of the mother and newborn 2013. Geneva: World Health Organization, 2013: 1-72.

67 McCarthy KJ, Blanc AK, Warren C, et al. Validating women's reports of antenatal and postnatal care received in Bangladesh, Cambodia and Kenya. BMJ Glob Health 2020;5:e002133. 